\title{
From the field into the lab: useful approaches to selecting species based on local knowledge
}

\author{
Adolfo Andrade-Cetto ${ }^{*}$ and Michael Heinrich ${ }^{2}$ \\ Laboratorio de Etnofarmacología, Departamento de Biología Celular, Facultad de Ciencias, Universidad Nacional Autónoma de México, Coyoacán, México \\ 2 Southern Cross Plant Science, Centre for Phytochemistry and Pharmacology, Southern Cross University, Lismore, NSW, Australia
}

Edited by:

Thomas Efferth, University of Mainz, Germany

\section{Reviewed by:}

Victor Kuete, University of Dschang, Cameroon

Hassan Elsubki Khalid, National Centre for Research Sudan, Sudan

\section{*Correspondence.}

Adolfo Andrade-Cetto, Laboratorio de Etnofarmacología, Departamento de Biología Celular, Facultad de Ciencias, Universidad Nacional Autónoma de México, Apartado Postal 70228, Coyoacán 04510, Ciudad de México, México.

e-mail:aac@.fciencias.unam.mx
Ethnopharmacological field studies are indispensable for identifying plants that can be selected for their pharmacological effects and chemical composition. Although the subjective interpretation of results by the researcher is crucial, quantitative data analysis is a useful tool to identify the most promising pharmacological plants. It has been stated that such semi-quantitative information increases the likelihood of finding promising ethnopharmacological leads, but so far no critical review has assessed what standards best meet the requirements of biomedical research. Systematic database searches using SCOPUS, Science Direct, Web of Knowledge, Science Citation Index, and Medline with the keywords "ethnobotany," "ethnopharmacology," "index," and "consensus" in research from the last 5 years form the basis of the current analysis, which identifies particularly useful tools like factor of informant consensus, fidelity level, use-value, and relative importance. A key feature for further field studies is that they should provide clear information on a range of topics like; detailed data of the importance of these resources within a culture, data of the uses of the species, how and where the plants are collected, drying and storage processes, preparation method, used doses, and administration. In addition, they must include a collection of records about how the people feel after the plant use, disappearance of specific symptoms and possible side effects.

Keywords: ethnobotany, ethnopharmacology, index, consensus, quantitative analysis, traditional medicine

\section{INTRODUCTION}

For ethnopharmacological experimental or clinical investigations, a relevant starting point is to understand who uses traditional medicine and how researchers in one of the many areas of biomedicine can get useful information about this use, which can guide the selection of medicinal plants for more detailed study.

Ethnopharmacology was initially defined as "the interdisciplinary scientific exploration of biological active agents traditionally employed or observed by man" (Holmsted and Bruhn, 1983). Another definition was provided by Etkin and Elisabetsky (2005): "By one, compelling logic, ethno- (Gr., culture or people) pharmacology (Gr., drug) is about the intersection of medical ethnography and the biology of therapeutic action." Based on the previous definitions, ethnopharmacology can be seen as the study of traditionally used, biologically active natural products, with the aim of understanding their therapeutic actions.

In ethnopharmacological experimental or clinical investigations, a crucial question is how the information about the use of natural products is obtained. One can expect that it comes directly from the people, who use the traditional medicine, important factor for non-written culture, in which the information is often passed down through oral communication. However, upon reviewing the ethnopharmacological literature describing local and traditional uses, one can see that the information does not always come directly from the people who use the remedy. Instead, the authors (lab-basedbiomedical-researchers, LBBRs), often obtain data from a secondary source, such as popular books, herbarium information, manufacturers, or other sources who are not the primary users of the plants. A distortion of the original information about the remedy use may occur because the interpretation may differ from the primary source.
It is therefore important to produce more information about the use of medicinal plants based on the original sources. An example is the garden spurge, for which a study highlighting the importance of certain taxa to treat skin conditions in Brisbane, Australia allowed the identification and development of the second most commonly mentioned species, Euphorbia peplus, as an interesting lead. It is now in Phase II clinical trials (Reuter et al., 2010). Additionally, when a source is used in a proper manner, it is a valid source of information. It is important to foster field-based ethnopharmacological studies as sources of new data, as highlighted by some authors (Edwards et al., 2005; Heinrich et al., 2009).

The aim of this work is to provide LBBRs with a small set of analytical tools to select quantitative data from the field. These tools are designed to help researchers analyze the results of structured interviews of people who use the traditional medicine. To use these tools, the researchers do not necessarily need detailed training in social or cultural sciences.

\section{SEARCH STRATEGY}

A search was performed in the following databases: Scopus ${ }^{1}$, Science Direct ${ }^{2}$, Web of Knowledge: Science Citation Index ${ }^{3}$, and Medline ${ }^{4}$. The keywords "ethnobotany," "ethnopharmacology," "index," and

\footnotetext{
${ }^{1}$ www.scopus.com

${ }^{2}$ Www.sciencedirect.com

${ }^{3}$ www.isiwebofknowledge.com

${ }^{4}$ www.ncbi.nlm.nih.gov/pubmed/
} 
"consensus" were used independently or in combination and results from the last 5 years were analyzed. The common tools used in the manuscripts and other useful tools are presented and an interpretation on how these tools can be used together is given.

\section{A REVIEW OF POSSIBLE APPROACHES}

As part of the data collection, some general requirements are important. The researcher needs to be precise about plant location (GPS), ethnographic background, sample size, and research design. It also needs to be decided if the focus of the research is on plant knowledge of healers, the general public, or people with a specific disease.

\section{OUANTITATIVE ETHNOBOTANICAL STUDIES}

A very comprehensive and critical comparative analysis of cultural importance indices was performed by Tardio and Pardo de Santayana (2008), but they did not assess the ethnopharmacological relevance of this information, as it relates to their use in prioritizing species for further biomedical research.

With the aim of providing clear explanations of methods in ethnobotany for use by the research community, Hoffman and Gallaher (2007) performed an Internet search using Google Scholar with the search terms "ethnobotany" and "use value." They recorded 12 specific methods and classified them into four categories; uses totaled or (researcher-tally), subjective allocation or (researcherscore), informant consensusor (informant tally), and informant consensusor (informant score) for the selection of the categories they use the proposal of Kvist et al. (1995) who used "tally" for methods that indiscriminately count every use cited and "score" for methods that sort uses into pre-determined hierarchical categories. In this work, they define relative cultural importance (RCI) indices as "quantitative measures designed to transform the complex, multidimensional concept of 'importance' into standardized and comparable numerical scales or values." They provide a set of 24 different formulas that can be applied to select plants used by man with any purpose.

\section{FACTOR OF INFORMANT CONSENSUS}

One of the most widely used tools is the factor of informant consensus $\left(F_{\text {ic }}\right)$. Its current form, proposed by Heinrich et al. (1998), was based on a similar but not identical definition first introduced by Trotter and Logan (1986).

$F_{\text {ic }}$ was used to identify plants of particular intercultural relevance and to agree on their use. It was originally used to analyze three populations with culturally different backgrounds in Mexico.

To use $F_{\text {ic }}$, it is necessary to classify illnesses into broad disease categories (several diseases based on the organ systems in one category). The original categories used by Heinrich et al. (1998) include conditions common to all five ethnic groups in Mexico: (1) gastrointestinal, (2) dermatological (mostly infections and subsequent inflammatory reactions), (3) respiratory, and (4) gynecological and andrological. They also compared illnesses deemed to be specific to one or two cultures such as "poisonous animal bites and stings" (Maya); ophthalmological illnesses (Maya and Zapotecs); and culture-bound syndromes (Nahua and Zapotecs). These authors used the formula.
The $F_{\text {ic }}$ was calculated as the number of use citations in each category $\left(N_{\mathrm{ur}}\right)$ minus the number of species used $(\mathrm{Nt})$, divided by the number of use citations in each category minus one (Heinrich et al., 1998):

$$
F_{\text {ic }}=\frac{N_{\mathrm{ur}}-N t}{N_{\mathrm{ur}}-1}
$$

$F_{\text {ic }}$ values range from 0.00 to 1.00 . High $F_{\text {ic }}$ values are obtained when only one or a few plant species are reported to be used by a high proportion of informants to treat a particular category, whereas low $F_{\text {ic }}$ values indicate that informants disagree over which plant to use. As a result of this analysis, it was possible to identify species of particular importance within a culture (intercultural) and to compare different cultures. Over the last 5 years this tool was used at least in 70 publications mainly to analyze the use of plant species in different ethnographic backgrounds; recent examples include Karousouand and Deirmentzoglou (2011) in Cyprus; Jacobo-Salcedo et al. (2011) in Mexico; Pandikumar et al. (2011) in India; Oliveira et al. (2010) in Brazil; Teklehaymanot and Giday (2009) in Ethiopia. $F_{\text {ic }}$ has also been used to analyze the use of animal species (Ferreira et al., 2009 and Upadhyay et al., 2010).

The main use of $F_{\text {ic }}$ is to select disease categories where there is consensus on the use of plants among the informants and to identify species with particular importance in a culture.

\section{FIDELITY LEVEL}

Of equal importance is the fidelity level (Fl), initially proposed Friedman et al. (1986) to analyze plant use among Bedouins in Israel. Fl was defined as the ratio between the number of informants who independently suggested the use of a species for the same major purpose and the total number of informants who mentioned the plant for any use.

In the Fl, $N p$ is the number of informants that reported a use of a plant species to treat a particular disease, and $N$ is the number of informants that used the plants as a medicine to treat any given disease (Friedman et al., 1986);

$$
\mathrm{Fl}=\frac{N p}{N \times 100}
$$

this index has been used in more than 50 manuscripts to identify the main use for one plant.

A limitation of this tool is that if a plant (A) has a low number of mentions (between two and three), the Fl can be high (100\%); in contrast a plant (B) with more mentions ( 15 or more) may have a lower Fl (84\%). This fact does not necessarily indicate more fidelity for plant (A) because in two uses there is $50 \%$ probability that the uses are different, whereas in 15 uses there is a greater probability of different uses and a low Fl (see Andrade-Cetto, 2009). To avoid this problem, the use-mentions (UM) factor was proposed. The UM is defined as the number of mentions for one plant given by all of the informants for a specific disease. The resulting UM value can be then correlated with the Fl to identify a plant with high fidelity. 


\section{USE-VALUE FOR ONE SPECIES}

The use-value (UV) index was used to calculate the citation of plants during interviews, proposed by Phillips and Gentry (1993a,b) and adapted by Albuquerque et al. (2007). It is calculated as follows:

$U V c=\sum U_{\text {is }} / n s$

where $U$ is the sum of the total number of use citations by all informants for a given species, divided by the total number of informants (ns). This method evaluates the relative importance (RI) of each medicinal species based on its relative use among informants.

This index is useful for the analysis of the use of a single species and to compare plants among the same sample (for example, see Aburjai et al., 2007).

\section{SPECIES USE-VALUE FOR ONE INFORMANT}

The species UV index was used to calculate the number of uses mentioned for species by one informant in different events (Phillips and Gentry, 1993a,b).

$$
U V_{\text {is }}=\left(\sum U_{\text {is }}\right) /\left(N_{\text {is }}\right)
$$

Where $U_{\text {is }}$ is the number of uses mentioned for species s by the informant and $N_{\text {is }}$ is the number of events in which the informant cites a use for species s.

This index is useful if a researcher goes into the field with one informant with the aim of collecting a specific species, but during the course of the field study, the informant mentions other plants; each time the informant stops and gives information about a species is an "event."

The expected score for each species is 1 , which means that, if a species was found four times, the informant mentioned the species four times for the same use.

\section{DISEASE-CONSENSUS INDEX}

With the aim of selecting species to treat a single, usually chronic disease, Andrade-Cetto et al. (2006) developed the disease-consensus index (DCI). For the application of the index, it is necessary to formulate a questionnaire with answers that can be evaluated in a binary way: 0 for no and 1 for yes. The questions must include personal knowledge about a specific species to treat the disease (see below). The index is a comparison based on mathematical aspects (limit theory), the ideal answers of informant reports (Cc) and the ideal answers for each species $(\mathrm{Vx})$.

It is calculated as follows: where;

- $(\mathrm{x})$ is any species.

- (mVxi) is the sum of the individual values obtained for one species within the community; it evaluates knowledge and mentions.

- $(\mathrm{mVx})$ is the statistical mean of the individual values for one species; it evaluates knowledge.

- Cc is the correlation coefficient, defined as the maximal number of informants who refer to a species; it evaluates mentions.

- $\mathrm{Pm}^{-0.1}$ is the compensation factor, and analyses the dispersion for one species, considering the mode of preparation and parts used.
Whit the formula:

$$
\mathrm{DCI}=\left(\sum_{i=1}^{\infty} \frac{\mathrm{Vxi}}{\mathrm{Cc}} \mathrm{mVx}\right) \mathrm{Pm}^{-0.1}
$$

In the original work, the following questions were used to calculate the index: (1) species name; (2) general plant description; (3) mode of preparation; (4) form of administration during a treatment; (5) "organoleptic" characteristics, such as flavor, odor, and texture; (6) main symptoms after the consumption of the species; (7) how often the species (in tea form) must be administered; (8) whether the person felt better after consuming the species; (9) general knowledge about the region of gathering or information about how to grow the species; (10) whether the patient recommended the species to other members of the community.

For the binary evaluation, only the alternatives "yes" or "no" are considered, not the main answer. For example, in Question 1, if the person knows the species name then the score for the index is one, no matter of what name $\mathrm{s} / \mathrm{he}$ gives the species (thus, one species may have more than one vernacular names). Likewise, for Question 2 the score is 1 if the person describes the species in some detail. If they cannot describe it, the score is 0 .

For the application of this methodology, some prerequisites are necessary: (1) the previous selection of the informants (normally those who present with the selected disease) and (2) the species need to be identified prior to the interviews.

This index is useful to select the plants most used by the informants (patients) to treat a single disease within a specific community.

\section{SIMPLE PERCENTAGE}

The simple percentage was employed by El-Hilal et al. (2003) in a study in a northern province of Morocco to highlight the relationship between medicinal plants and main therapeutic indications selected according to interviews. The sum of all the indications was defined as 100\%, and the percentage of each single indication was then calculated. This analysis is useful to select the main diseases in a study area.

\section{RELATIVE IMPORTANCE INDEX}

In an effort to understand how species were introduced to the pharmacopeia of northern South America, Bennett and Prance (2000) proposed the RI index. Originally, they compiled data on medicinal plants from their own studies and from selected publications. The RI was then calculated for each medicinal plant based on the normalized number of body systems (BS) that one species affects and the pharmacological proprieties $(\mathrm{PH})$ that are attributed to the same species. The result can have a maximum value of 2 ; the final value is multiplied by 50 to convert to a scale of 100 .

For example, if a species $\mathrm{X}$ is employed to treat $12 \mathrm{BS}$, the maximum any species can obtain is a normalized value of $B S$ of $12 / 12=1$. If $\mathrm{X}$ has a $\mathrm{pH}$ value of $15 \mathrm{pH}$, while the most versatile species $\mathrm{Y}$ has a $\mathrm{pH}$ value of $\mathrm{Y}$, then the normalized $\mathrm{pH}$ value of $\mathrm{X}$ is $15 / 17=1.88$. To convert to a scale of 100 , the combined values $(1+1.88)$ are multiplied by 50 , giving an RI of $2.88 \times 50=94.1$. The index was adapted by Albuquerque and Oliveira (2007) to test the hypothesis that the RI can be explained by the species habitat or its status (spontaneous or cultivated, endemic or not endemic) as follows: 


\section{$\mathrm{RI}=\mathrm{NCS}+\mathrm{NP}, \mathrm{NCS}=\mathrm{NCSS} / \mathrm{NSCSV}, \mathrm{NP}=\mathrm{NPS} / \mathrm{NPSV}$}

where NCS is the number of BS, NCSS the number of BS treated by a given species, NSCSV the total number of BS treated by the most versatile species (the most versatile species are those that have the greatest number of medicinal properties), NP the number of pharmacological properties, NPS the number of properties attributed to a species and NPSV the total number of properties attributed to the most versatile species.

The main use of this index is to measure the potential of a given medicinal species, including its capacity to treat more than one health problem. It has been used to analyze data presented previously in the literature.

\section{DISCUSSION}

Over the last 5 years, more than 800 ethnobotanical studies have quantitatively analyzed data; nearly $90 \%$ of these studies are about medicinal plants.

The objective of a field-based study (the "ethno" in "ethnopharmacology") often is the selection of species for further pharmacological studies, but it should not be limited to this goal. While such field studies should be stand-alone contribution, from the perspective of an experimental pharmacologist or a phytochemist, a range of key outcomes of such field studies are desirable.

The analysis of the data obtained in these studies requires the interpretation of the researcher. The main analysis regarding the traditional use of the species to treat a disease requires subjective interpretation and thus cannot be measured quantitatively. If we considered, in one

\section{REFERENCES}

Aburjai, T., Hudaib, M., Tayyem, R., Yousef, M., and Qishawi M. (2007). Ethnopharmacological survey of medicinal herbs in Jordan, the Ajloun Heights region. Ethiopia J. Ethnopharmacol. 110, 294-304.

Albuquerque, U. P., Medeiros, P. M., Almeida, A. L., Monteiro, J. M., LinsNeto, E. M. F., Melo, J. G., and Santos, J. P. (2007). Medicinal plants of the caatinga (semi-arid) vegetation of NE Brazil: a quantitative approach. J. Ethnopharmacol. 114, 325-354.

Albuquerque, U. P., and Oliveira, R. F. (2007). Is the use-impact on native caatinga species in Brazil reduced by the high species richness of medicinal plants? J. Ethnopharmacol. 113, 156-170.

Andrade-Cetto,A. (2009). Ethnobotanical study of the medicinal plants from Tlanchinol, Hidalgo, México. J. Ethnopharmacol. 122, 163-171.

Andrade-Cetto, A., Becerra-Jiménez, J., Martínez-Zurita, E., Ortega-Larrocea, M.P., and Heinrich, M. (2006). Diseaseconsensus index as a tool of selecting potential hypoglycemic plants in Chikindzonot, Yucatan, México. J. Ethnopharmacol. 107, 199-204.

Bennett, B., and Prance, G. T. (2000). Introduced plants in the indigenous

pharmacopoeia of northern South

hand, that LBBRs, in general does not have an ethnological background and thus the subjective data interpretation can be a problem and on the other hand, reliable and repeatable data about the plants are needed for further pharmacological studies, we propose the use of quantitative analysis in field studies performed by LBBRs.

The factor of informant consensus is particularly useful to select the categories of diseases for which the species are traditionally used. By applying this tool, researchers can identify the main diseases in a community grouped by category. In addition to $F_{\text {ic }}$, Fl can be used to select single plants. This combination allows the selection of one or more species used in the main categories. These analyses can be completed using, simple percentage and UM.

The UV index allows the identification of a species within a sample without taking into account its use (Category); this is useful in the comparison between species for the whole sample. While the species UV is useful during field studies with a single informant, the score for one species can be compared between several informants.

If the objective of the analysis is to identify plants used to treat a specific chronic disease, then the DCI is the best option.

Finally, the RI index can help the researcher analyze previous field studies already reported in the literature.

With the goal of obtaining information about the traditional use of plants, the indices presented here provide a set of tools for the quantitative analysis of an ethnopharmacological field study.

\section{ACKNOWLEDGMENTS}

This work was partially supported by the DGAPA, PAPIIT project IN228510, and CONACyT CB 079910.

bedouins in the Negev desert, Israel. J. Ethnopharmacol. 16, 275-287. America. Econ. Bot. 54, 90-102.

Edwards, S., Nebel, S., and Heinrich, M. (2005). Questionnaire surveys: methodological and epistemological problems for field-based ethnopharmacologists J. Ethnopharmacol. 100, 30-36.

El-Hilal, J., Hmammouchi, M., and Badiaa, L. (2003). Ethnobotanical studies and economic evaluation of medicinal plants in Taounate province (Northern Morocco). J. Ethnopharmacol. 86, 149-158

Etkin, N., and Elisabetsky, E. (2005). Seeking a transdisciplinary and culturally germane science: the future of ethnopharmacology. J. Ethnopharmacol. 100 23-26.

Ferreira, F. S., Brito1, S. V., Ribeiro, S. C., Saraiva, A. A., Almeida 1, W. O., and Alves, R. R. (2009). Animalbased folk remedies sold in public markets in Crato and Juazeiro do Norte, Ceará, Brazil. BMC Complement Altern. Med. 9, 17. doi: 10.1186/1472-6882-9-17

Friedman, J., Yaniv, Z., Dafni, A., and Palevitch, D. (1986). A preliminary classification of the healing potential of medicinal plants, based on a rational analysis of an ethnopharmacological field survey among
Heinrich, M., Ankli, A., Frei, B., Weimann, C., and Sticher, O. (1998). Medicinal plants in Mexico: healers' consensus and cultural importance. Soc. Sci. Med. 47, 1859-1871.

Heinrich, M., Edwards, S., Moerman, D., and Leonti, M. (2009). Ethnopharmacological field studies: A critical assessment of their conceptual basis and methods. J. Ethnopharmacol. 124, 1-17.

Hoffman, B., and Gallaher, T. (2007). Importance Indices in Ethnobotany. Ethnobotany Res. Appl. 5, 201-218.

Holmstedt, B., and Bruhn, J. (1983). Ethnopharmacology - a challenge. J. Ethnopharmacol. 8, 251-256.

Jacobo-Salcedo, M. R., Alonso-Castro, A. J., and Zarate-Martinez, A. (2011). Folk medicinal use of fauna in Mapimi, Durango, México. J. Ethnopharmacol. 133, 902-906.

Karousouand, R., and Deirmentzoglou, S. (2011). The herbal market of Cyprus: traditional links and cultural exchanges. J. Ethnopharmacol. 133, 191-203.

Kvist, P. Andersen, M. Hesselsoe, M., and Vanclay, J. (1995). Estimating use-values and relative importance of Amazonian flood plain trees and forests to local inhabitants. Commonw. For. Rev. 74, 293-300.

Oliveira, E., Torres, D., Brooks, S., and Alves, R. (2010). The medicinal animal markets in the metropolitan region of Natal City, northeastern Brazil. J. Ethnopharmacol. 130, 54-60.

Pandikumar, P., Chellappandian, M., Mutheeswaran, S., and Ignacimuthu, S. (2011). Consensus of local knowledge on medicinal plants among traditional healers in Mayiladumparai block of Theni District, Tamil Nadu, India. J. Ethnopharmacol. 134, 354-362.

Phillips, O., and Gentry, A. H. (1993a). The useful plants of Tambopata, Peru: I. Statistical hypotheses tests with a newquantitative technique. Econ. Bot. 47, 15-32.

Phillips, O., and Gentry, A. H. (1993b). The useful plants of Tambopata, Peru: II. Statistical hypotheses tests with a new quantitative technique. Econ. Bot. 47, 33-43.

Reuter, J., Wölfle, U., Korting, H.C., and Schempp, C. (2010). Which plant for which skin disease? Part 2: dermatophytes, chronic venous insufficiency, photoprotection, actinic keratoses, vitiligo, hair loss, cosmetic indications. J. Ger. Soc. Dermatol. 8, 866-873. 
Tardío, J., and Pardo de Santayana, M. (2008). Cultural importance indices: a comparative analysis based on the useful wild plants of southern cantabria (Northern Spain).Econ. Bot.62, 24-39. Teklehaymanot, T., and Giday, M. (2009). Quantitative ethnobotany of medicinal plants used by Kara and Kwego semi-pastoralist people in lower Omo River Valley, Debub Omo Zone, Southern Nations, Nationalities and Peoples Regional State, Ethiopia J. Ethnopharmacol. 130, 76-84.
Trotter, R. T., and Logan, M. H. (1986). "Informant consensus: a new approach for identifying potentially effective medicinal plants," in Plants in Indigenous Medicine and Diet: Biobehavioral Approaches, ed. N. L. Etkin,(New York: Redgrave Publishing Company), 91-112.

Upadhyay, B., Singh, K. P., and Kumar, A. (2010). Ethno-veterinary uses and informants consensus factor of medicinal plants of Sariska region, Rajasthan, India. J. Ethnopharmacol. 133, 14-25.
Conflict of Interest Statement: The authors declare that the research was conducted in the absence of any commercial or financial relationships that could be construed as a potential conflict of interest.

Received: 16 February 2011; accepted: 28 March 2011; published online: 09 April 2011.

Citation: Andrade-Cetto A and Heinrich $M$ (2011) From the field into the lab: useful approaches to selecting species based on local knowledge. Front. Pharmacol. 2:20. doi: 10.3389/fphar.2011.00020

This article was submitted to Frontiers in Ethnopharmacology, a specialty of Frontiers in Pharmacology.

Copyright (c) 2011 Andrade-Cetto and Heinrich. This is an open-access article subject to a non-exclusive license between the authors and Frontiers Media SA, which permits use, distribution and reproduction in other forums, provided the original authors and source are credited and other Frontiers conditions are complied with. 